\title{
Nitrogen and Phosphorus Uptake Dynamics in Tropical Cerrado Woodland Streams
}

\author{
Nícolas Reinaldo Finkler ${ }^{1}$ (D), Flavia Tromboni ${ }^{2, *}$, Iola Gonçalves Boëchat ${ }^{3}{ }^{(0)}$, Björn Gücker ${ }^{3}$ (D) \\ and Davi Gasparini Fernandes Cunha ${ }^{1}$ (iD \\ 1 Department of Hydraulic and Sanitation Engineering, São Carlos School of Engineering, \\ University of São Paulo, São Carlos, São Paulo CEP 13560-590, Brazil; nicolas.finkler@gmail.com (N.R.F.); \\ davig@sc.usp.br (D.G.F.C.) \\ 2 Global Water Center and Department of Biology, University of Nevada, Reno, NV 89557, USA \\ 3 Department of Geosciences, Federal University of São João del-Rei, São João del-Rei, \\ Minas Gerais CEP 36301-360, Brazil; iboechat@ufsj.edu.br (I.G.B.); guecker@ufsj.edu.br (B.G.) \\ * Correspondence: flavia.tromboni@gmail.com; Tel.: +1-775-420-7335
}

Received: 15 July 2018; Accepted: 31 July 2018; Published: 14 August 2018

\begin{abstract}
Pollution abatement through phosphorus and nitrogen retention is a key ecosystem service provided by streams. Human activities have been changing in-stream nutrient concentrations, thereby altering lotic ecosystem functioning, especially in developing countries. We estimated nutrient uptake metrics (ambient uptake length, areal uptake rate, and uptake velocity) for nitrate $\left(\mathrm{NO}_{3}-\mathrm{N}\right)$, ammonium $\left(\mathrm{NH}_{4}-\mathrm{N}\right)$, and soluble reactive phosphorus (SRP) in four tropical Cerrado headwater streams during 2017, through whole-stream nutrient addition experiments. According to multiple regression models, ambient SRP concentration was an important explanatory variable of nutrient uptake. Further, best models included ambient $\mathrm{NO}_{3}-\mathrm{N}$ and water velocity (for $\mathrm{NO}_{3}-\mathrm{N}$ uptake metrics), dissolved oxygen (DO) and canopy cover (for $\mathrm{NH}_{4}-\mathrm{N}$ ); and $\mathrm{DO}$, discharge, water velocity, and temperature (for SRP). The best kinetic models describing nutrient uptake were efficiency-loss ( $\mathrm{R}^{2}$ from $0.47-0.88$ ) and first-order models ( $\mathrm{R}^{2}$ from $\left.0.60-0.85\right) . \mathrm{NO}_{3}-\mathrm{N}, \mathrm{NH}_{4}-\mathrm{N}$, and SRP uptake in these streams seemed coupled as a result of complex interactions of biotic $\mathrm{P}$ limitation, abiotic $\mathrm{P}$ cycling processes, and the preferential uptake of $\mathrm{NH}_{4}-\mathrm{N}$ among $\mathrm{N}$-forms. Global change effects on these tropical streams, such as temperature increase and nutrient enrichment due to urban and agricultural expansion, may have adverse and partially unpredictable impacts on whole-stream nutrient processing.
\end{abstract}

Keywords: low-order streams; nutrient retention; self-purification capacity; Tracer Additions for Spiraling Curve Characterization; tropical water bodies

\section{Introduction}

Streams are biogeochemical active systems that alter the amount and chemical form of nutrients and organic matter transported from their catchments to downstream systems [1,2]. Stream nitrogen $(\mathrm{N})$ and phosphorus $(\mathrm{P})$ retention is considered an important ecosystem service as it controls the export of these nutrients to downstream rivers, lakes, estuaries, and ultimately oceans, mitigating the negative consequences of cultural eutrophication [3,4]. Human activities have considerably altered nutrient availability, mobility, and distribution in freshwater systems [5-7], affecting ecosystem-wide nutrient and organic matter dynamics [8], and thus freshwater ecosystem functioning.

Variability in $\mathrm{N}$ and $\mathrm{P}$ retention rates and efficiency has been associated with primary production [9-11], hydrology and stream geomorphology [12,13], carbon availability [14-16], and nutrient limitation and availability [11,17-19]. Several studies have assessed how environmental 
concentration influences the uptake of a particular nutrient [20-23]. Some empirical studies have considered multi-elemental effects and dynamics [24,25], but they are rare in tropical regions (but see [19]). In addition, some authors have explored correlations between $\mathrm{N}$ and $\mathrm{P}$ uptake in streams including measurements of either nitrate-nitrogen $\left(\mathrm{NO}_{3}-\mathrm{N}\right)[26,27]$ or ammonium-nitrogen $\left(\mathrm{NH}_{4}-\mathrm{N}\right)[28,29]$, but rarely both.

Few studies have measured the uptake of soluble reactive phosphorus (SRP), $\mathrm{NO}_{3}-\mathrm{N}$, and $\mathrm{NH}_{4}-\mathrm{N}$ together (e.g., [30]), and those suggested that colimitation by $\mathrm{N}$ and $\mathrm{P}$ can occur in streams [19]. There is increasing evidence of the predominance of colimitation and interactions among multiple elements in both aquatic and terrestrial ecosystems (e.g., [8,31-33]). The assessment of nutrient limitation is important because the dynamics and downstream transport of a limiting nutrient differ from those of a non-limiting one [19]. Understanding the implications of elemental interactions in nutrient retention studies is essential for the management of water quality in streams and downstream ecosystems.

Studies of nutrient dynamics in the tropics are rare, but especially important in developing countries, which experience high rates of population growth and rural-urban migration [34]. In these countries, $90-95 \%$ of all sewage, and $70 \%$ of industrial waste are still not treated [35]. The lack of sewage treatment and increasing urbanization in catchments can significantly alter in-stream nutrient concentrations $[11,36]$. Other impacts occurring in tropical regions are related to deforestation of pristine vegetation for pastoral and agricultural uses. These are associated with decreases in stream nutrient concentrations in some tropical catchments, due to the depletion of soil organic matter stocks, but with increases in other catchments, probably due to fertilizer use (see literature review in [37]). Some studies have reported strong correlations between uptake rates and ambient nutrient availability [38-40], while others found no influence of background nutrient concentrations on retention [41], highlighting the need for further research.

Nutrient uptake can be affected by nutrient enrichment in three different ways. First, the biotic community can track nutrient availability, resulting in constant uptake velocity and linear increases in areal uptake rate with increasing nutrient concentration [2,17]. Second, biological processes can be less efficient or saturated under higher ambient concentrations. Saturation or loss of efficiency causes uptake velocity to decline and areal uptake rate to increase in a curvilinear fashion with increasing concentration $[11,42,43]$. Third, the uptake of a particular nutrient can covary with the relative availability of another nutrient $[18,44]$. For example, $\mathrm{P}$ enrichment can lead to $\mathrm{N}$ limitation and stimulate $\mathrm{N}$ uptake, and vice-versa.

The aforementioned studies usually examined nutrient enrichment associated with land use gradients (e.g., $[18,22,42,45])$. Land use can also influence other variables, such as water discharge, light availability, sediment inputs, and ecosystem metabolism, all of which directly or indirectly influence nutrient uptake $[46,47]$. Here, we investigated a gradient in nutrient concentration across forested, tropical headwater streams. We studied whole-stream $\mathrm{NO}_{3}-\mathrm{N}, \mathrm{NH}_{4}-\mathrm{N}$, and SRP uptake across a gradient of ambient stream nutrient concentrations to expand our knowledge about interactions between $\mathrm{N}$ and $\mathrm{P}$ cycling and how these interactions are affected by nutrient availability in tropical streams. We studied four streams located in the Brazilian Cerrado savanna biome to address the following research questions: (1) How does nutrient uptake vary across a gradient of ambient nutrient availability? (2) How do the absolute and relative availability of one nutrient influence the uptake of the other nutrient? (3) Are there positive or negative relationships between $\mathrm{NO}_{3}-\mathrm{N}, \mathrm{NH}_{4}-\mathrm{N}$, and SRP uptake rates? (4) Are nutrient uptake rates higher or lower than those reported for temperate streams in the literature? In general, we hypothesized a higher demand for $\mathrm{NH}_{4}-\mathrm{N}$ than for SRP and $\mathrm{NO}_{3}-\mathrm{N}$, because $\mathrm{NH}_{4}-\mathrm{N}$ is the $\mathrm{N}$-form preferentially assimilated by biota [45]. More enriched streams were expected to be less retentive than less enriched ones, showing lower areal uptake rates and uptake velocities. Finally, we hypothesized that none of the studied streams would exhibit saturation conditions (i.e., significant fits to the Michaelis-Menten kinetic model) because of the absence of chronical nutrient inputs. 


\section{Methods}

We carried this study out in four low-order Cerrado streams (discharge less than $20 \mathrm{~L} \cdot \mathrm{s}^{-1}$ ) located in São Carlos and Brotas (São Paulo, Southeastern Brazil; Table 1; Figure 1), and in each of them, we selected a representative $100-\mathrm{m}$ long stream reach. The Cerrado biome (Brazilian woodland savannah) is the second-largest South American biome, rich in springs and low-order lotic networks, which contribute to 8 of the 12 large Brazilian river basins [48]. The sampling sites Espraiado (ESP) and Broa (BRO) were located in relatively preserved basins with extensive and dense riparian vegetation, natural substrate, and restricted access. Canchim (CAN) was located in the EMBRAPA Pecuária Sudeste experimental farm, presenting a preserved riparian zone and natural substrata. Mineirinho tributary (TBM) had an urban drainage basin with fragmented riparian vegetation and advanced erosive features; however, it did not receive domestic or industrial effluents and its headwaters were preserved. The studied streams differed in water depth (from 0.04 to $0.48 \mathrm{~m}$ ), and were relatively narrow with wetted widths ranging from 0.50 to $1.20 \mathrm{~m}$.

Table 1. Characterization of the streams used in this study with geographic coordinate, drainage area, and general reach characterization.

\begin{tabular}{llll}
\hline Stream (Site Code) & $\begin{array}{l}\text { Geographic } \\
\text { Coordinates }\end{array}$ & $\begin{array}{l}\text { Drainage } \\
\text { Area }\left(\mathbf{k m}^{2}\right)\end{array}$ & General Characteristics \\
\hline Broa (BRO) & $\begin{array}{l}22^{\circ} 11^{\prime} 40.93^{\prime \prime} \mathrm{S} \\
47^{\circ} 53^{\prime} 55.78^{\prime \prime} \mathrm{W}\end{array}$ & 2.68 & $\begin{array}{l}\text { Natural vegetation, forested and dense riparian } \\
\text { zone, natural substrate, located upstream from a } \\
\text { natural wetland }\end{array}$ \\
\hline Canchim (CAN) & $\begin{array}{l}21^{\circ} 57^{\prime} 54.69^{\prime \prime} \mathrm{S} \\
47^{\circ} 50^{\prime} 38.02^{\prime \prime} \mathrm{W}\end{array}$ & 1.17 & $\begin{array}{l}\text { Natural vegetation, forested and dense riparian } \\
\text { zone, natural substrate, located next to the spring }\end{array}$ \\
\hline Espraiado (ESP) & $\begin{array}{l}21^{\circ} 58^{\prime} 46.75^{\prime \prime} \mathrm{S} \\
47^{\circ} 52^{\prime} 23.11^{\prime \prime} \mathrm{W}\end{array}$ & 2.49 & $\begin{array}{l}\text { Natural vegetation, forested and dense riparian } \\
\text { zone, meandric channel, natural substrate }\end{array}$ \\
\hline $\begin{array}{l}\text { Mineirinho } \\
\text { tributary (TBM) }\end{array}$ & $\begin{array}{l}22^{\circ} 00^{\prime} 12.78^{\prime \prime} \mathrm{S} \\
47^{\circ} 55^{\prime} 40.82^{\prime \prime} \mathrm{W}\end{array}$ & 0.82 & $\begin{array}{l}\text { Relatively degraded vegetation, natural } \\
\text { substrate, presence of erosive features and } \\
\text { pluvial runoff }\end{array}$ \\
\hline
\end{tabular}

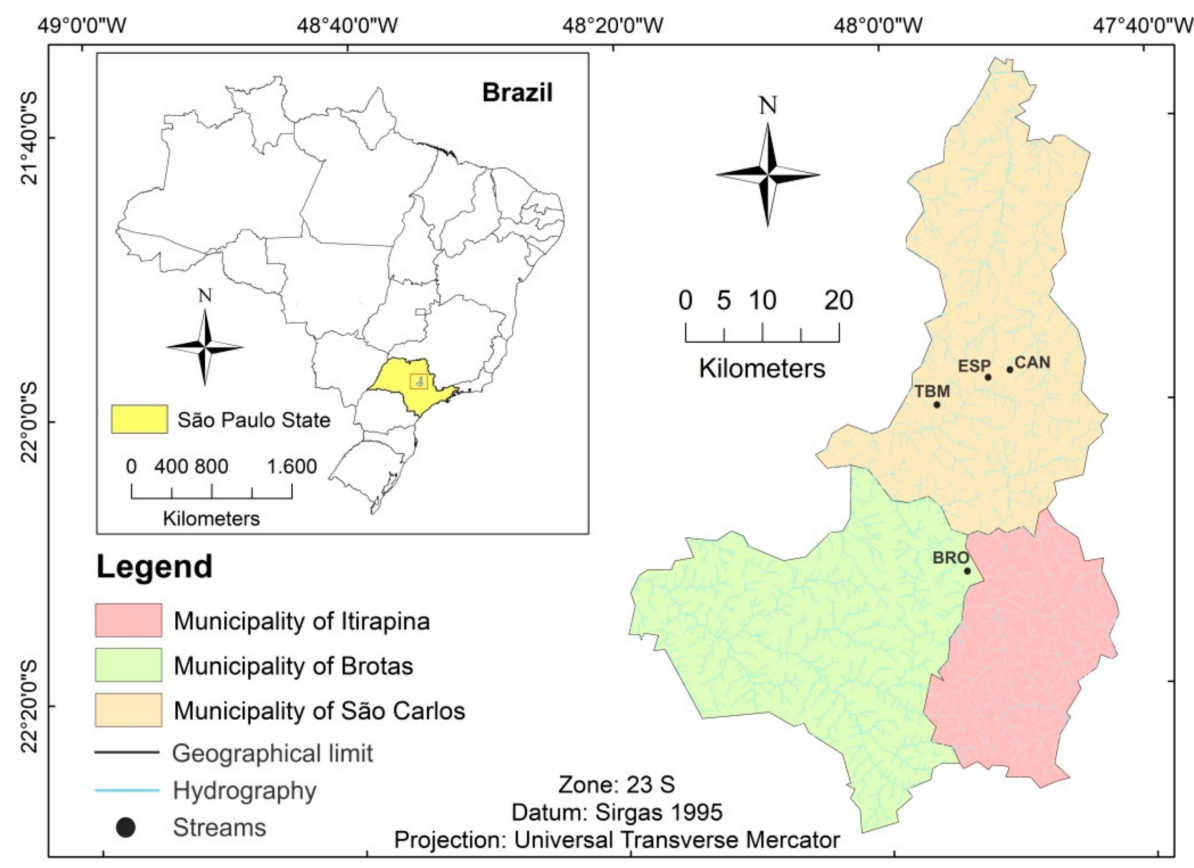

Figure 1. Sampling locations BRO (Broa), CAN (Canchim), ESP (Espraiado), and TBM (Mineirinho River tributary) in southeast Brazil. 
We ran four sets of nutrient additions over the year 2017 (January, April, July, and October), encompassing wet and dry seasons. All addition experiments were carried out under base-flow conditions. We used the tracer additions for spiraling curve characterization (TASCC) approach described in the literature [49] to estimate uptake metrics from pulsed nutrient additions. This method allows determining total (tot-dyn), added (add-dyn), and ambient $(\mathrm{amb})$ uptake metrics. We calculated the uptake metrics uptake length $\left(\mathrm{S}_{\mathrm{W}}\right)$, areal uptake rate $(\mathrm{U})$, and uptake velocity $\left(\mathrm{V}_{\mathrm{f}}\right)$ for different $\mathrm{N}$ and $\mathrm{P}$ forms according to the nutrient spiraling concept [2].

To all stream reaches, we simultaneously added $\mathrm{NO}_{3}-\mathrm{N}$ as $\mathrm{NaNO}_{3}, \mathrm{NH}_{4}-\mathrm{N}$ as $\mathrm{NH}_{4} \mathrm{Cl}$, and SRP as $\mathrm{K}_{2} \mathrm{HPO}_{4}$ as bioavailable reactive tracers to characterize nutrient dynamics, and chloride $\left(\mathrm{Cl}^{-}\right)$as $\mathrm{NaCl}$ as a conservative tracer to characterize stream hydrodynamics. The added mass of conservative tracer was calculated prior to each experiment to increase in-stream electrical conductivity (EC) at measurable, but moderate levels (i.e., 5-10-fold of background EC), while the added mass of nutrients was calculated to raise instream concentrations to up to 2-5-fold of background concentrations [49]. The ratio of $\mathrm{NH}_{4}-\mathrm{N}$ to $\mathrm{NO}_{3}-\mathrm{N}$ in nutrient additions ranged from 0.25 to 0.62 . We dissolved all salts in a $5 \mathrm{~L}$ bucket with stream water and then poured the solution carefully into the stream at the top of the experimental reach over one minute (i.e., as a slug). Electric conductivity was measured at $10 \mathrm{~s}$ intervals over the experiment using a multiparameter probe at the downstream end of the reach (Model HI 9829, HANNA Instruments, Woonsocket, RI, USA). At this station, we took water samples over the full pulse, with sampling frequency ranging from $15 \mathrm{~s}$ to $5 \mathrm{~min}$ as a function of EC rate of rise or decline, resulting in 21-26 samples per experiment, in order to obtain a well-characterized breakthrough curve. Immediately before the additions, we collected three water samples to determine nutrient background (i.e., ambient) concentrations. Water samples were filtered immediately upon collection (GF/C Glass Microfiber Membranes, $0.45 \mu \mathrm{m}$, Whatman International, Kent, UK) and frozen at $-18{ }^{\circ} \mathrm{C}$ until analysis. All water samples were analyzed within a maximum of two weeks after collection.

Stream water variables (i.e., $\mathrm{pH}, \mathrm{EC}$, temperature, and dissolved oxygen) were measured using the multiparameter probe (HANNA HI 9829, HANNA Instruments, Woonsocket, RI, US). All nutrient concentrations were determined via colorimetry using a Hach DR 4000V spectrophotometer (Hach Environmental, Loveland, CO, USA). The method used for $\mathrm{NH}_{4}-\mathrm{N}$ was based on the literature [50], modified for a $7 \mathrm{~mL}$ sample volume, and the ones used for SRP and $\mathrm{NO}_{3}-\mathrm{N}$ followed the literature [51]. Dissolved organic carbon (DOC) was analyzed using a Shimadzu SSM 5000 TOC analyzer (Shimadzu Corp., Norcross, GA, USA) the combustion method.

We also estimated the canopy cover percentage in each stream reach using a concave densitometer (Forestry Suppliers Inc., Jackson, MS, USA) following the literature [52,53]. Dilution gauging was used to measure stream discharge at the downstream ends of each experimental reach [54]. Air temperature and total precipitation were obtained from a nearby meteorological station belonging to the Brazilian National Institute of Meteorology (INMET, www.inmet.gov.br), located in São Carlos (code: 83726, latitude $-21^{\prime} 96^{\circ}$, longitude $-47^{\prime} 86^{\circ}$, altitude $856 \mathrm{~m}$ ).

Differences among ambient nutrient concentrations across streams were tested using a Kruskal-Wallis test due to the non-normality of data. Spearman rank correlation was initially used to explore general correlations between uptake metrics and in-stream variables (i.e., physical, chemical, and hydraulic variables). We performed general regression analyses using uptake metrics as dependent variables, and as independent variables, those selected as important uptake drivers based on literature research (e.g., [17,30,55-57]). Among the selected variables, we excluded those that were autocorrelated (i.e., through Spearman rank tests). The remaining set of variables was then submitted to backward stepwise selection to obtain the multiple linear regression models that best described relationships between uptake metrics and environmental variables. We considered data from all streams and samplings together in regression models; therefore, the generated models incorporated typical spatial and seasonal variability of the study region, which we considered to be representative of tropical 
Cerrado woodlands. All data were previously $\ln (x+1)$ transformed, in order to achieve normally distribution, as a prerequisite of linear regression models.

We also adjusted our experimental data to three kinetic models to evaluate the uptake dynamic as function of nutrient concentration. Here, we used the TASCC total dynamics metrics (i.e., total areal uptake rate as $U_{\text {tot-dyn, }}$ and total dynamic nutrient concentration as $C_{\text {tot-dyn }}$ [49]). The Michaelis-Menten kinetic model (M-M) represents saturation in uptake when nutrient availability greatly exceeds uptake via biological processes [18]. The efficiency-loss (ELS) kinetic model suggests that nutrient uptake is less efficient at high concentrations, even if saturation is not reached [20]. The first-order kinetic model (FTO) assumes that there is no saturation of uptake rate, and that the relationship between uptake rate and nutrient concentration is positive and linear. This model is often assumed to represent nutrient uptake in pristine, nutrient-limited settings.

Linear regression $(p<0.05)$ was used to determine the regression fit with the ELS and FTO models. Dependent and independent variables were $\ln (x+1)$ transformed to satisfy the normality of residuals assumption of this method and reduce the effects of extreme values. Fits to the $\mathrm{M}-\mathrm{M}$ model were processed using untransformed data [20]. Saturation was considered if there was a significant adjustment to the $\mathrm{M}-\mathrm{M}$ model and the calculated $\mathrm{K}_{\mathrm{m}}$ (i.e., the half-saturation constant) was within the range of experimental nutrient concentrations. To fit the data to all three models, we used least squares regressions with the Levenberg-Marquardt algorithm. We conducted statistical analyses using Statistica 10 (Statsoft, Tulsa, OK, USA) and fits to kinetics models and graphical representation were performed with Origin 2017 (OriginLab Corp., Northampton, MA, USA).

\section{Results}

\subsection{General Characterization of the Streams}

Total annual precipitation between December 2016 and November 2017 was 1042 mm. Monthly precipitation varied between 0 and $178 \mathrm{~mm}$, with $72 \%$ of total rainfall concentrated between December 2016 and May 2017. Stream discharge varied between 2.0 and $19.2 \mathrm{~L} \cdot \mathrm{s}^{-1}$ considering all sites and sampling dates (Table 2). Water velocities ranged from 0.04 to $0.60 \mathrm{~m} \cdot \mathrm{s}^{-1}$. Ranges for EC were $4-32 \mu \mathrm{S} \cdot \mathrm{cm}^{-1}$, for water temperature $15-23^{\circ} \mathrm{C}$, for $\mathrm{pH} 4.4-6.5$, and for dissolved oxygen $5.4-8.6 \mathrm{mg} \cdot \mathrm{L}^{-1}$. The riparian zones were dense in all sites with canopy cover varying between $77 \%$ and $98 \%$, with ESP as the most forested site (92-98\%).

Table 2. Ambient physical and chemical characteristics of studied streams. DOC (dissolved organic carbon), DO (dissolved oxygen), EC (electric conductivity), T (water temperature), Q (stream discharge), $\mathrm{v}$ (water velocity), $\mathrm{H}$ (depth), w (wetted width), and CC (percentage of canopy cover).

\begin{tabular}{ccccccccccc}
\hline Site & $\begin{array}{c}\mathbf{D O C} \\
\left(\mathbf{m g} \cdot \mathbf{L}^{-\mathbf{1}}\right)\end{array}$ & $\begin{array}{c}\mathbf{D O} \\
\left(\mathbf{m g} \cdot \mathbf{L}^{-\mathbf{1}}\right)\end{array}$ & $\mathbf{p H}$ & $\begin{array}{c}\mathbf{E C} \\
\left(\boldsymbol{\mu S} \cdot \mathbf{c m}^{-\mathbf{1}}\right)\end{array}$ & $\mathbf{T}\left(^{\circ} \mathbf{C}\right)$ & $\begin{array}{c}\mathbf{Q} \\
\left(\mathbf{L} \cdot \mathbf{s}^{-\mathbf{1}}\right)\end{array}$ & $\begin{array}{c}\mathbf{v} \\
\left(\mathbf{m} \cdot \mathbf{s}^{-\mathbf{1}}\right)\end{array}$ & $\mathbf{H}(\mathbf{c m})$ & $\mathbf{w}(\mathbf{m})$ & $\mathbf{C C}(\mathbf{\%})$ \\
\hline BRO & $1.3-3.8$ & $6.9-7.9$ & $4.4-5.3$ & $4-6$ & $16.5-21.6$ & $7.5-11.2$ & $0.10-0.30$ & $4-16$ & $0.6-1.0$ & $90-98$ \\
ESP & $1.4-3.3$ & $5.5-7.4$ & $4.9-5.6$ & $11-19$ & $15-20.6$ & $5.0-19.2$ & $0.06-0.26$ & $5-48$ & $0.5-0.6$ & $93-98$ \\
TBM & $1.2-2.4$ & $7.1-8.3$ & $5.8-6.1$ & $19-28$ & $17.5-22.8$ & $3.4-6.9$ & $0.10-0.60$ & $1-5$ & $1.0-1.2$ & $77-91$ \\
CAN & $1.6-2.7$ & $7.5-8.5$ & $5.9-6.5$ & $21-32$ & $15-20.5$ & $2.0-4.0$ & $0.04-0.25$ & $1-8$ & $0.9-1.1$ & $92-97$ \\
\hline
\end{tabular}

Background dissolved organic carbon concentration did not differ significantly among streams $(p=0.94)$ and was always lower than $4.0 \mathrm{mg} \cdot \mathrm{L}^{-1}$ (Table 2). Concentrations of $\mathrm{NO}_{3}-\mathrm{N}$ and $\mathrm{NH}_{4}-\mathrm{N}$ (Table 3) also did not differ significantly among streams ( $p=0.47$ and $p=0.57$, respectively), with annual medians of the four studied streams ranging from $37-457 \mu \mathrm{g} \cdot \mathrm{L}^{-1}$ for $\mathrm{NO}_{3}-\mathrm{N}$, and $5-36 \mu \mathrm{g} \cdot \mathrm{L}^{-1}$ for $\mathrm{NH}_{4}-\mathrm{N}$. However, there were significant differences in SRP concentrations among streams $(p<0.005)$, with annual medians ranging between 1 and $23 \mu \mathrm{g} \cdot \mathrm{L}^{-1}$. Accordingly, we classified our streams using background SRP concentrations to facilitate the visualization of SRP limitation effects on nutrient uptake. Therefore, all graphs and tables are presented in the following order of SRP concentration (Table 3): $\mathrm{BRO}<\mathrm{ESP}<\mathrm{TBM}<\mathrm{CAN}$. 
Table 3. Ambient uptake length $\left(\mathrm{S}_{\mathrm{w} \text {-amb }} ; \mathrm{m}\right)$, areal uptake rate $\left(\mathrm{U}_{\mathrm{amb}}\right)$, uptake velocity $\left(\mathrm{V}_{\mathrm{f}-\mathrm{amb}} ; \mathrm{mm} \cdot \mathrm{min}^{-1}\right)$, and nutrient concentration $\left(\mathrm{C}_{\mathrm{amb}} ; \mu \mathrm{g} \cdot \mathrm{L}^{-1}\right)$ during nutrient additions in four tropical streams for each nutrient form expressed as annual medians (minimum-maximum values). SRP-soluble reactive phosphorus.

\begin{tabular}{|c|c|c|c|c|c|c|c|c|c|c|c|c|}
\hline \multirow[b]{2}{*}{ Site } & \multicolumn{4}{|c|}{$\mathrm{NH}_{4}-\mathrm{N}$} & \multicolumn{4}{|c|}{$\mathrm{NO}_{3}-\mathrm{N}$} & \multicolumn{4}{|c|}{ SRP } \\
\hline & $\mathrm{S}_{\mathrm{w}-\mathrm{amb}}(\mathrm{m})$ & $\underset{\left(\mu \mathrm{g} \cdot \mathrm{m}^{-2} \cdot \mathrm{min}^{-1}\right)}{\mathrm{U}_{\mathrm{amb}}}$ & $\begin{array}{c}V_{\mathrm{f}-\mathrm{amb}} \\
\left(\mathrm{mm} \cdot \mathrm{min}^{-1}\right)\end{array}$ & $\underset{\left(\mu \mathrm{g} \cdot \mathrm{L}^{-1}\right)}{\mathrm{C}_{\mathrm{amb}}}$ & $\mathrm{S}_{\mathrm{w}-\mathrm{amb}}(\mathrm{m})$ & $\underset{\left(\mu \mathrm{g} \cdot \mathrm{m}^{-2} \cdot \mathrm{min}^{-1}\right)}{\mathrm{U}_{\mathrm{m} b}}$ & $\frac{V_{f-a m b}}{\left(m m \cdot \min ^{-1}\right)}$ & $\underset{\left(\mu \mathrm{g} \cdot \mathrm{L}^{-1}\right)}{\mathrm{C}_{\mathrm{amb}}}$ & $S_{\mathrm{w}-\mathrm{amb}}(\mathrm{m})$ & $\underset{\left(\mu \mathrm{g} \cdot \mathrm{m}^{-2} \cdot \mathrm{min}^{-1}\right)}{\mathrm{U}_{\mathrm{amb}}}$ & $\begin{array}{c}\mathrm{V}_{\mathrm{f}-\mathrm{amb}} \\
\left(\mathrm{mm} \cdot \mathrm{min}^{-1}\right)\end{array}$ & $\begin{array}{c}\mathrm{C}_{\mathrm{amb}} \\
\left(\mu \mathrm{g} \cdot \mathrm{L}^{-1}\right)\end{array}$ \\
\hline All sites & $\begin{array}{c}70 \\
(32-253)\end{array}$ & $\begin{array}{c}75 \\
(5-593)\end{array}$ & $\begin{array}{c}7.5 \\
(0.8-68)\end{array}$ & $\begin{array}{c}6 \\
(5-36)\end{array}$ & $\begin{array}{c}150 \\
(7-418)\end{array}$ & $\begin{array}{c}593 \\
(56-46,373)\end{array}$ & $\begin{array}{c}2.4 \\
(0.5-123)\end{array}$ & $\begin{array}{c}315 \\
(37-457)\end{array}$ & $\begin{array}{c}144 \\
(55-559)\end{array}$ & $\begin{array}{c}35 \\
(9-149)\end{array}$ & $\begin{array}{c}4.3 \\
(0.5-17)\end{array}$ & $\begin{array}{c}13 \\
(1-23)\end{array}$ \\
\hline BRO & $\begin{array}{c}44 \\
(32-59) \\
\end{array}$ & $\begin{array}{c}251 \\
(121-593)\end{array}$ & $\begin{array}{c}24.3 \\
(16-68)\end{array}$ & $\begin{array}{c}8 \\
(5-24)\end{array}$ & $\begin{array}{c}63 \\
(8-418)\end{array}$ & $\begin{array}{c}8829 \\
(714-46,373) \\
\end{array}$ & $\begin{array}{c}32.0 \\
(2-123) \\
\end{array}$ & $\begin{array}{c}357 \\
(177-382) \\
\end{array}$ & $\begin{array}{c}64 \\
(55-105) \\
\end{array}$ & $\begin{array}{c}53 \\
(10-79) \\
\end{array}$ & $\begin{array}{c}16.0 \\
(7-17)\end{array}$ & $\begin{array}{c}3 \\
(1-5) \\
\end{array}$ \\
\hline ESP & $\begin{array}{c}90 \\
(57-152) \\
\end{array}$ & $\begin{array}{c}112 \\
(41-274) \\
\end{array}$ & $\begin{array}{c}9 \\
(7-25) \\
\end{array}$ & $\begin{array}{c}8 \\
(5-36) \\
\end{array}$ & $\begin{array}{c}249 \\
(139-313) \\
\end{array}$ & $\begin{array}{c}1131 \\
(667-2038) \\
\end{array}$ & $\begin{array}{c}4.0 \\
(3.6-5.6) \\
\end{array}$ & $\begin{array}{c}322 \\
(183-407) \\
\end{array}$ & $\begin{array}{c}138 \\
(42-154) \\
\end{array}$ & $\begin{array}{c}87 \\
(60-149) \\
\end{array}$ & $\begin{array}{c}8.0 \\
(7-12) \\
\end{array}$ & $\begin{array}{c}11 \\
(8-13) \\
\end{array}$ \\
\hline TBM & $\begin{array}{c}116 \\
(62-172) \\
\end{array}$ & $\begin{array}{c}13 \\
(10-20)\end{array}$ & $\begin{array}{c}3 \\
(1.6-3)\end{array}$ & $\begin{array}{c}6 \\
(5-6.7)\end{array}$ & $\begin{array}{c}194 \\
(95-351)\end{array}$ & $\begin{array}{c}264 \\
(56-519)\end{array}$ & $\begin{array}{c}1.3 \\
(1-3)\end{array}$ & $\begin{array}{c}142 \\
(37-392) \\
\end{array}$ & $\begin{array}{c}283 \\
(156-560)\end{array}$ & $\begin{array}{c}12 \\
(9-15)\end{array}$ & $\begin{array}{c}1.0 \\
(0.6-1)\end{array}$ & $\begin{array}{c}13 \\
(8-15)\end{array}$ \\
\hline CAN & $\begin{array}{c}193 \\
(35-253)\end{array}$ & $\begin{array}{c}8 \\
(76-96)\end{array}$ & $\begin{array}{c}0.9 \\
(0.7-6)\end{array}$ & $\begin{array}{c}10 \\
(5-12)\end{array}$ & $\begin{array}{c}132 \\
(131-373)\end{array}$ & $\begin{array}{c}313 \\
(155-328)\end{array}$ & $\begin{array}{c}1.0 \\
(0.5-1.6)\end{array}$ & $\begin{array}{c}316 \\
(202-457)\end{array}$ & $\begin{array}{c}201 \\
(77-391)\end{array}$ & $\begin{array}{c}24 \\
(10-36)\end{array}$ & $\begin{array}{c}1.0 \\
(0.5-1.5)\end{array}$ & $\begin{array}{c}21 \\
(18-23)\end{array}$ \\
\hline
\end{tabular}




\subsection{Nutrient Uptake Metrics and Kinetics}

Among streams, annual median uptake lengths ranged from 44-193 $\mathrm{m}$ for $\mathrm{NH}_{4}-\mathrm{N}, 63-249 \mathrm{~m}$ for $\mathrm{NO}_{3}-\mathrm{N}$, and $64-283 \mathrm{~m}$ for SRP (Table 3). Annual median areal uptake rates ranged from 8-251 $\mu \mathrm{g} \cdot \mathrm{m}^{-2} \cdot \mathrm{min}^{-1}$ among streams for $\mathrm{NH}_{4}-\mathrm{N}, 264-8829 \mu \mathrm{g} \cdot \mathrm{m}^{-2} \cdot \mathrm{min}^{-1}$ for $\mathrm{NO}_{3}-\mathrm{N}_{\text {, }}$ and $12-87 \mu \mathrm{g} \cdot \mathrm{m}^{-2} \cdot \mathrm{min}^{-1}$ for SRP. Annual median uptake velocities ranged from 0.9-24.3, 1.0-32.0, and 1.0-16.0 $\mathrm{mm} \cdot \mathrm{min}^{-1}$ for $\mathrm{NH}_{4}-\mathrm{N}, \mathrm{NO}_{3}-\mathrm{N}$, and SRP, respectively (Table 3).

Spearman rank correlations used for parameter selection are available as Supplementary Material (Table S1). For multiple regression models, we used $\mathrm{U}_{\mathrm{amb}}$ and $\mathrm{V}_{\mathrm{f} \text {-amb }}$ as the dependent variables

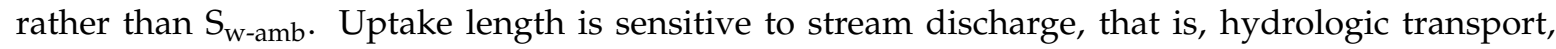
and accordingly, we found only weak relationships between $S_{\mathrm{w}-\mathrm{amb}}$ and other environmental variables. Therefore, we considered $\mathrm{U}_{\mathrm{amb}}$ and $\mathrm{V}_{\mathrm{f} \text {-amb }}$ to be more representative of biogeochemical nutrient demand. All multiple linear regression models were significant $\left(p<0.05\right.$, adjusted $\mathrm{R}^{2} 0.48$ to 0.89 ; Table 4). In these multiple regressions, there was a negative influence of SRP concentrations on $\mathrm{N}$ uptake rates and velocity. $\mathrm{NO}_{3}-\mathrm{N}$ concentration had a positive effect on $\mathrm{NO}_{3}-\mathrm{N}$ uptake rate. Furthermore, SRP concentrations had a negative influence on SRP uptake velocity, while $\mathrm{NH}_{4}-\mathrm{N}$ concentrations did not affect any nutrient uptake metrics. More forested streams appeared to be more $\mathrm{NH}_{4}-\mathrm{N}$ retentive than more open-canopy ones, as we found a strong positive relationship between the percentage of canopy cover and $\mathrm{NH}_{4}-\mathrm{N}$ uptake rate. $\mathrm{DO}$ concentrations had a negative effect on $\mathrm{NH}_{4}-\mathrm{N}$ uptake, while DO, water temperature, and velocity had negative effects on SRP retention.

Table 4. Multiple linear regressions of ambient uptake metrics $\left(\mathrm{U}_{\mathrm{amb}}\right.$ and $\left.\mathrm{V}_{\mathrm{f} \text {-amb }}\right)$ as function of environmental variables. All data used were $\ln$-transformed $[\ln (x+1)]$.

\begin{tabular}{|c|c|c|c|c|c|}
\hline Dependent Variable & Independent Variable & B & SE & $p$-Value & Adjusted $R^{2}$ \\
\hline \multirow{3}{*}{$\mathrm{U}_{\mathrm{amb}} \mathrm{NO}_{3}-\mathrm{N}\left(\mu \mathrm{g} \cdot \mathrm{m}^{-2} \cdot \mathrm{min}^{-1}\right)$} & Intercept & 2.20 & 3.50 & 0.54 & \multirow{3}{*}{0.482} \\
\hline & $\mathrm{SRP}_{\mathrm{amb}}$ & -1.33 & 0.52 & $2 \times 10^{2}$ & \\
\hline & $\mathrm{NO}_{3 \mathrm{amb}}$ & 1.14 & 0.57 & $3 \times 10^{2}$ & \\
\hline \multirow{4}{*}{$\mathrm{U}_{\mathrm{amb}} \mathrm{NH}_{4}-\mathrm{N}\left(\mu \mathrm{g} \cdot \mathrm{m}^{-2} \cdot \mathrm{min}^{-1}\right)$} & Intercept & -12.25 & 13.15 & 0.37 & \multirow{4}{*}{0.824} \\
\hline & $\mathrm{SRP}_{\mathrm{amb}}$ & -1.64 & 0.24 & $3 \times 10^{5}$ & \\
\hline & DO & -4.68 & 1.52 & $1 \times 10^{2}$ & \\
\hline & $\% \mathrm{CC}$ & 6.61 & 2.65 & $3 \times 10^{2}$ & \\
\hline \multirow{5}{*}{$\mathrm{U}_{\mathrm{amb}} \operatorname{SRP}\left(\mu \mathrm{g} \cdot \mathrm{m}^{-2} \cdot \mathrm{min}^{-1}\right)$} & Intercept & 24.28 & 4.20 & $1 \times 10^{4}$ & \multirow{5}{*}{0.816} \\
\hline & DO & -4.60 & 1.13 & $2 \times 10^{3}$ & \\
\hline & $\mathrm{Q}$ & 0.47 & 0.21 & $4 \times 10^{2}$ & \\
\hline & $\mathrm{V}$ & -3.08 & 0.99 & $1 \times 10^{2}$ & \\
\hline & $\mathrm{T}$ & -3.85 & 0.92 & $1 \times 10^{3}$ & \\
\hline \multirow{3}{*}{$\mathrm{V}_{\mathrm{f}-\mathrm{amb}} \mathrm{NO}_{3}-\mathrm{N}\left(\mathrm{mm} \cdot \mathrm{min}^{-1}\right)$} & Intercept & 5.31 & 0.97 & $2 \times 10^{4}$ & \multirow{3}{*}{0.508} \\
\hline & $\mathrm{SRP}_{\mathrm{amb}}$ & -1.12 & 0.36 & $1 \times 10^{2}$ & \\
\hline & $\mathrm{v}$ & -5.31 & 2.20 & $3 \times 10^{2}$ & \\
\hline \multirow{3}{*}{$\mathrm{V}_{\mathrm{f}-\mathrm{amb}} \mathrm{NH}_{4}-\mathrm{N}\left(\mathrm{mm} \cdot \mathrm{min}^{-1}\right)$} & Intercept & 13.03 & 3.00 & $1 \times 10^{3}$ & \multirow{3}{*}{0.731} \\
\hline & $\mathrm{SRP}_{\mathrm{amb}}$ & -1.35 & 0.22 & $8 \times 10^{4}$ & \\
\hline & DO & -3.74 & 1.39 & $2 \times 10^{2}$ & \\
\hline \multirow{5}{*}{$\mathrm{V}_{\mathrm{f}-\mathrm{amb}} \mathrm{SRP}\left(\mathrm{mm} \cdot \mathrm{min}^{-1}\right)$} & Intercept & 21.34 & 3.10 & $3 \times 10^{5}$ & \multirow{5}{*}{0.889} \\
\hline & $\mathrm{SRP}_{\mathrm{amb}}$ & -1.05 & 0.12 & $2 \times 10^{6}$ & \\
\hline & $\mathrm{DO}$ & -3.71 & 0.84 & $1 \times 10^{3}$ & \\
\hline & $\mathrm{T}$ & -2.97 & 0.73 & $2 \times 10^{3}$ & \\
\hline & $\mathrm{v}$ & -2.77 & 0.79 & $4 \times 10^{3}$ & \\
\hline
\end{tabular}

For all streams, there was a better fit of total dynamic uptake rate $\left(\mathrm{U}_{\text {tot-dyn }}\right)$ with total dynamic nutrient concentration $\left(\mathrm{C}_{\text {tot-dyn }}\right)$ than with total dynamic uptake velocity $\left(\mathrm{V}_{\mathrm{f} \text {-tot-dyn }}\right)$ (Figure 2 , Supplementary Tables S2-S4). For uptake rate (U) kinetics (Table 5), there was no significant fit to the M-M model, suggesting no saturation of uptake rates. In general, the relationship between $U$ and nutrient concentration did not appear to follow a specific model across streams. For U, site BRO 
had a better fit to the ELS model for all nutrients $\left(\mathrm{R}^{2}=0.12,0.47\right.$, and 0.79 for $\mathrm{NO}_{3}-\mathrm{N}, \mathrm{NH}_{4}-\mathrm{N}$, and $\mathrm{SRP}$, respectively) than to $\mathrm{M}-\mathrm{M}$ and FTO models. Site ESP showed a better fit to the ELS model for $\mathrm{NO}_{3}-\mathrm{N}$ $\mathrm{U}\left(\mathrm{R}^{2}=0.70\right)$ and SRP $\mathrm{U}\left(\mathrm{R}^{2}=0.88\right)$, while $\mathrm{NH}_{4}-\mathrm{N}$ U followed the FTO model $\left(\mathrm{R}^{2}=0.80\right)$. Site TBM followed the FTO model for $\mathrm{NO}_{3}-\mathrm{N} \mathrm{U}$ and SRP $U\left(\mathrm{R}^{2}=0.60\right.$ and $\mathrm{R}^{2}=0.83$, respectively), while for $\mathrm{NH}_{4}-\mathrm{N}$ U, the ELS model showed the best fit $\left(\mathrm{R}^{2}=0.68\right)$. The FTO model showed the best fit in site CAN for $\mathrm{NH}_{4}-\mathrm{N}$ and SRP $U\left(\mathrm{R}^{2}=0.70\right.$ and $\mathrm{R}^{2}=0.85$, respectively), while there was a better fit to the ELS model for $\mathrm{NO}_{3}-\mathrm{N} \mathrm{U}\left(\mathrm{R}^{2}=0.73\right)$ in $\mathrm{CAN}$.

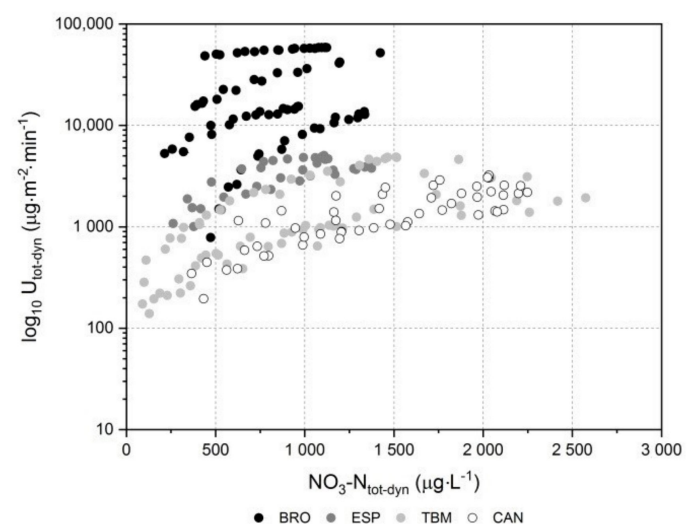

(a)

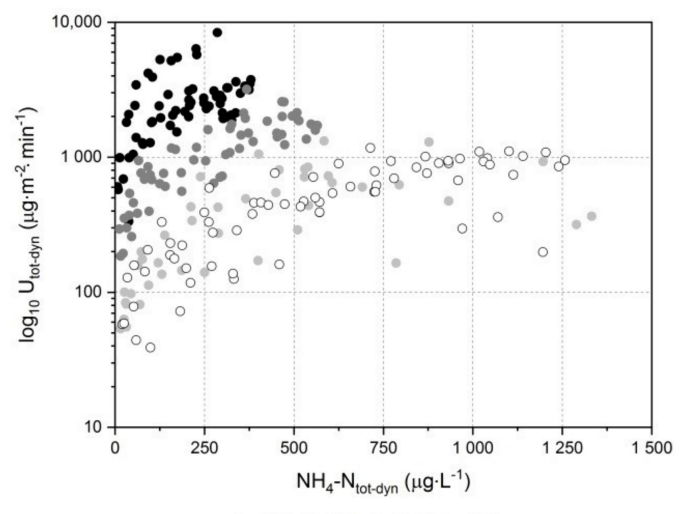

- BRo - ESP - TBM O CAN

(c)

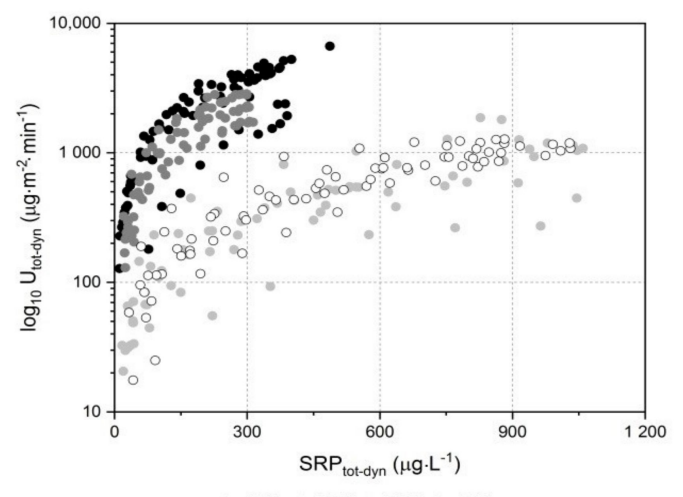

- bro - esp - tBM ○ can

(e)

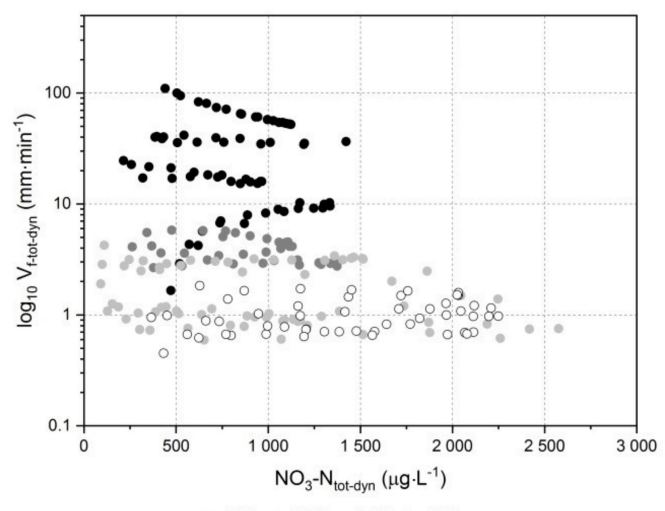

(b)

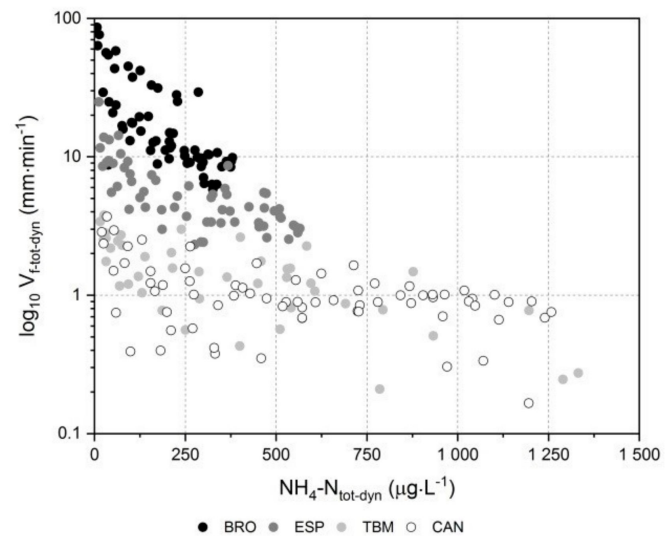

(d)

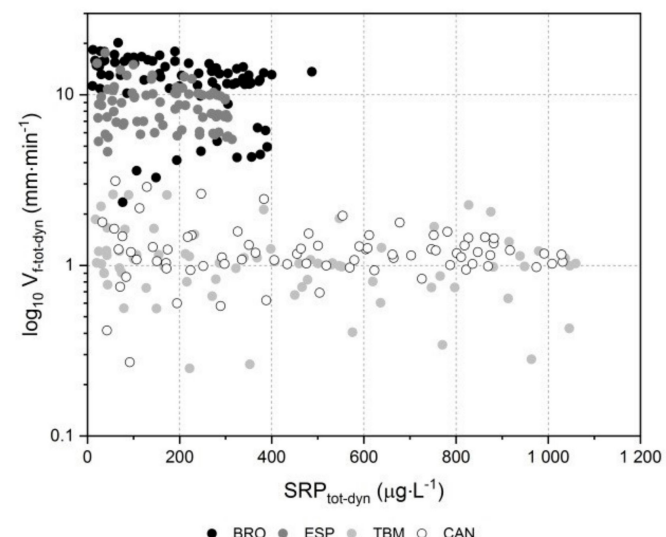

(f)

Figure 2. Relationships between $\mathrm{U}_{\text {tot-dyn }}, \mathrm{V}_{\mathrm{f} \text {-ot-dyn, }}$ and $\mathrm{C}_{\text {tot-dyn }}$ for $\mathrm{NO}_{3}-\mathrm{N}, \mathrm{NH}_{4}-\mathrm{N}$, and $\mathrm{SRP}$ during the period from January to October 2017. (a) $\mathrm{U}_{\text {tot-dyn }}$ versus $\mathrm{C}_{\text {tot-dyn }}$ for $\mathrm{NO}_{3}-\mathrm{N}$; (b) $\mathrm{V}_{\mathrm{f} \text {-ot-dyn }}$ versus $\mathrm{C}_{\text {tot-dyn }}$ for $\mathrm{NO}_{3}-\mathrm{N}$; (c) $\mathrm{U}_{\text {tot-dyn }}$ versus $\mathrm{C}_{\text {tot-dyn }}$ for $\mathrm{NH}_{4}-\mathrm{N}$; (d) $\mathrm{V}_{\mathrm{f} \text {-ot-dyn }}$ versus $\mathrm{C}_{\text {tot-dyn }}$ for $\mathrm{NH}_{4}-\mathrm{N}$;

(e) $\mathrm{U}_{\text {tot-dyn }}$ versus $\mathrm{C}_{\text {tot-dyn }}$ for SRP; (f) $\mathrm{V}_{\mathrm{f} \text {-ot-dyn }}$ versus $\mathrm{C}_{\text {tot-dyn }}$ for SRP. 
Table 5. Kinetic model adjustments between $V_{\text {ft-tot-dyn }}$ versus $C_{\text {tot-dyn }}$ and $U_{\text {tot-dyn }}$ versus $C_{\text {tot-dyn }}$ for each stream considering all data collected from January to October 2017. In any case, $\mathrm{R}^{2}$ is represented. ELS: efficiency-loss; FTO: first-order; M-M: Michaelis-Menten.

\begin{tabular}{|c|c|c|c|}
\hline \multirow{2}{*}{ Stream } & \multicolumn{3}{|c|}{ Best Kinetic Model $\left(\mathrm{R}^{2}\right)$ for $\mathrm{V}_{\mathrm{ft} \text {-tot-dyn }}$ versus $\mathrm{N}_{\text {tot-dyn }}$} \\
\hline & $\mathrm{NO}_{3}-\mathrm{N}$ & $\mathrm{NH}_{4}-\mathrm{N}$ & SRP \\
\hline $\mathrm{BRO}$ & - & M-M (0.653) & - \\
\hline ESP & FTO (0.649) & ELS (0.658) & - \\
\hline TBM & - & FTO $(0.453)$ & - \\
\hline CAN & - & ELS (0.364) & - \\
\hline \multirow{2}{*}{ Stream } & \multicolumn{3}{|c|}{ Best Kinetic Model $\left(R^{2}\right)$ for $U_{\text {tot-dyn }}$ versus $N_{\text {tot-dyn }}$} \\
\hline & $\mathrm{NO}_{3}-\mathrm{N}$ & $\mathrm{NH}_{4}-\mathrm{N}$ & SRP \\
\hline $\mathrm{BRO}$ & - & ELS (0.465) & ELS (0.787) \\
\hline ESP & ELS (0.697) & FTO (0.795) & ELS (0.881) \\
\hline TBM & FTO (0.596) & ELS (0.679) & FTO (0.831) \\
\hline CAN & ELS (0.733) & FTO (0.702) & FTO (0.854) \\
\hline
\end{tabular}

\section{Discussion}

Forested heterotrophic headwater streams, such as the streams we studied [58], typically have a considerable nutrient retention capacity (i.e., short $S_{\mathrm{w} \text {-amb }}$ and high $\mathrm{U}_{\mathrm{amb}}$ and $\mathrm{V}_{\mathrm{f} \text {-amb }}[10,14]$ ). Several studies have also reported substantial $\mathrm{NO}_{3}-\mathrm{N}, \mathrm{NH}_{4}-\mathrm{N}$, and SRP retention in pristine tropical headwater streams $[40,41,57,59]$. The authors of [41] attributed high rates of $\mathrm{NH}_{4}-\mathrm{N}$ uptake in tropical headwater streams to microbial activity, which is probably energy-limited because decomposition of terrestrial leaf litter is rapid, dissolved organic $C$ is strongly retained by mineral soils, and light availability limits primary production in these streams. Further, the authors of [57] highlighted the importance of structural complexity, which increases residence time of solutes and available instream surface area, both important variables for aquatic microbial biofilms and thus for ammonium uptake in tropical Cerrado savanna streams. High nutrient uptake in tropical streams appears to be related to these processes rather than to water column nutrient concentrations, or just high temperature increasing microbial metabolism. In general, the tropical Cerrado woodland stream sites studied here corresponded well with this pattern; nutrient uptake lengths were short and uptake velocities and rates for all nutrient forms were high compared with literature data from temperate streams, especially for $\mathrm{N}$ forms. However, the metrics reported here were similar to those reported from other tropical streams of similar size (Table 6; representing a literature review of peer-reviewed articles on nutrient spiraling in tropical streams published prior to July 2018 and available at Web of Science and Science Direct). For a comparison with temperate systems, we used data from a literature review [14], which presented uptake metrics from 52 published studies, mostly of them carried out in temperate zones. 
Table 6. Compilation of $\mathrm{NO}_{3}-\mathrm{N}, \mathrm{NH}_{4}-\mathrm{N}$, and SRP uptake results from natural streams sites located in different regions. The values are shown as range of metrics (min-max) when available; otherwise, the mean values are represented. Data from Ensign and Doyle [14] are uptake metrics from first-order streams.

\begin{tabular}{|c|c|c|c|c|c|c|}
\hline Nutrient & Stream & Location & $S_{w-a m b}(m)$ & $\underset{\left(\mu \mathrm{g} \cdot \mathrm{m}^{-2} \cdot \min ^{-1}\right)}{\mathrm{U}_{\mathrm{amb}}}$ & $\begin{array}{c}\mathrm{V}_{\mathrm{f}-\mathrm{amb}} \\
\left(\mathrm{mm} \cdot \mathrm{min}^{-1}\right)\end{array}$ & Reference \\
\hline \multirow{8}{*}{$\mathrm{NO}_{3}-\mathrm{N}$} & - & Temperate & $101-478$ & $5.8-19.1$ & $0.8-4.2$ & [14] \\
\hline & Barra Pequena & \multirow{7}{*}{ Tropical } & $467-499$ & 94 & - & [19] \\
\hline & Q Bisley & & 1192 & 16 & 1.92 & [59] \\
\hline & - & & $315-8480$ & - & $0.13-0.94$ & [40] \\
\hline & BRO & & $6(8-418)$ & $8829(714-46,373)$ & $32(2-123)$ & \multirow{4}{*}{ This study } \\
\hline & ESP & & $249(139-313)$ & 1131 (667-2038) & $4(3.6-5.6)$ & \\
\hline & TBM & & $194(95-351)$ & $264(56-519)$ & $1.3(1-3)$ & \\
\hline & CAN & & $132(131-373)$ & $313(155-328)$ & $1(0.5-1.6)$ & \\
\hline \multirow{9}{*}{$\mathrm{NH}_{4}-\mathrm{N}$} & - & Temperate & $23-275$ & $5.3-52.6$ & $2.5-17.0$ & [14] \\
\hline & Barra Pequena & \multirow{8}{*}{ Tropical } & $138-501$ & $5.45-14.2$ & - & [19] \\
\hline & Q. Bisley & & $15-26$ & $33-42$ & 87.3 & [59] \\
\hline & R. Cipó & & - & 1000 & - & [13] \\
\hline & - & & - & - & $0.3-8.5$ & [41] \\
\hline & BRO & & $44(32-59)$ & $251(121-593)$ & $24.3(16-68)$ & \multirow{4}{*}{ This study } \\
\hline & ESP & & $90(57-152)$ & $112(41-274)$ & $9(7-25)$ & \\
\hline & TBM & & $116(62-172)$ & $13(10-20)$ & $3(1.6-3)$ & \\
\hline & CAN & & $193(35-253)$ & $8(76-96)$ & $0.9(0.7-6)$ & \\
\hline \multirow{7}{*}{ SRP } & - & Temperate & 24-161 & $3.4-15.7$ & $1.5-6.6$ & [14] \\
\hline & Barra Pequena & \multirow{6}{*}{ Tropical } & $461-1065$ & $6.5-63$ & - & [19] \\
\hline & Carapa & & - & $950-1150$ & - & [60] \\
\hline & BRO & & $64(55-105)$ & $53(10-79)$ & $16(7-17)$ & \multirow{4}{*}{ This study } \\
\hline & ESP & & $138(42-154)$ & $86(60-149)$ & $8(7-12)$ & \\
\hline & TBM & & $283(156-560)$ & $12(9-15)$ & $1(0.6-1)$ & \\
\hline & CAN & & $201(77-391)$ & $24(10-36)$ & $1(0.5-1.5)$ & \\
\hline
\end{tabular}

Low SRP concentrations and predominantly high ambient molar DIN:SRP ratios (annual median of 24 to 279 across streams) would predict short uptake lengths and high uptake velocities for SRP compared with DIN forms. The authors of [61] suggested P limitation in streams at molar DIN:SRP ratios higher than 20, and consequently, a stoichiometric dominance of $\mathrm{P}$ uptake over $\mathrm{N}$ uptake. However, $\mathrm{NH}_{4}-\mathrm{N}$ presented the shortest $\mathrm{S}_{\mathrm{w} \text {-amb }}$ and the greatest $\mathrm{V}_{\mathrm{f} \text {-amb }}$ in our study, indicating high retention and demand for $\mathrm{NH}_{4}-\mathrm{N} . \mathrm{NO}_{3}-\mathrm{N}$ had the longest $\mathrm{S}_{\mathrm{w}-\mathrm{amb}}$ among all nutrient forms, indicating lowest retention relative to hydrologic transport of this nutrient form, and SRP had the lowest $\mathrm{V}_{\mathrm{f}-\mathrm{amb}}$, suggesting lowest demand.

Above a molar Redfield ratio of N:P of 16:1 [62], $\mathrm{P}$ is expected to limit algal growth ( $\mathrm{N}$ is in excess), and below this ratio there is an $\mathrm{N}$ deficit (P is in excess). The authors of [58] presented molar TN:TP ratios of 44 to 195 in the stream water for the same reaches we studied here, which would suggest $P$ limitation. The lack of a relationship between SRP uptake metrics and DIN concentrations further supports P limitation as the increased availability of $\mathrm{N}$ should not facilitate SRP uptake if phosphorus is the primary limiting nutrient. Surprisingly, the slope of the relationship of N uptake rates and velocities, as well as $\mathrm{U}_{\mathrm{DIN}} \mathrm{U}_{\mathrm{SRP}}$ ratios, with SRP concentrations was persistently negative in our study. We hypothesize that this negative relationship may have been caused by the rather special $P$ geochemistry of the studied region (see discussion in subsequent paragraphs), that is, anaerobic sediment conditions stimulating $P$ release, but at the same time negatively affecting aerobic assimilatory $\mathrm{N}$ uptake. In conclusion, the rather complex relationships between $\mathrm{NO}_{3}-\mathrm{N}, \mathrm{NH}_{4}-\mathrm{N}$, and SRP uptake in our streams appear to be due to the interplay of biotic $\mathrm{P}$ limitation, abiotic $\mathrm{P}$ cycling processes, and the preferential uptake of $\mathrm{NH}_{4}-\mathrm{N}$ among $\mathrm{N}$-forms. 
While inorganic $\mathrm{N}$ uptake appeared to be driven mostly by biotic process in our study, because it was dominated by $\mathrm{NO}_{3}$-uptake (i.e., higher areal $\mathrm{NO}_{3}-\mathrm{N}$ than $\mathrm{NH}_{4}-\mathrm{N}$ uptake rates), that does not present relevant abiotic uptake processes, SRP uptake might be related to abiotic processes. The relative importance of biotic and abiotic mechanisms for $\mathrm{P}$ retention can vary depending on $\mathrm{P}$ loading rates and in-stream conditions. At low levels of P loading, biotic uptake exceeds sorption [63], while under elevated $\mathrm{P}$ inputs, sorption can become a predominant mechanism depending on stream geochemistry [64]. Experimental whole-stream SRP additions and laboratory assays based on sediment sorption isotherms have shown that abiotic factors can dominate P retention in streams $[60,65,66]$.

Abiotic P uptake occurs through sorption, which includes both adsorption to surfaces of cationic minerals and precipitation with electrolytes $[67,68]$, and could be high in small streams, in which the sediment surface area to water volume ratio is high and contact time of stream water with sediments is long [69]. Fe and $\mathrm{Al}$ complexed with organic matter can be responsible for P sorption [68], however, we did not find relationships between SRP uptakes and dissolved organic C. In sediments dominated by Fe minerals, such as those present in Cerrado streams [70], reduction of soluble ferrous oxyhydroxide compounds results in the formation of $\mathrm{P}$ sorption sites. This reduction is the result of facultative organisms using ferric iron as an electron acceptor during their metabolic process in absence of oxygen. On the other hand, a reduced sediment surface layer can allow for considerable $\mathrm{PO}_{4}{ }^{3-}$ release from $\mathrm{Fe}(\mathrm{III})$ oxide, whereas an oxidized sediment surface represents an efficient geochemical barrier for sediment $P$ release [71].

Phosphorus uptake can be primarily governed by temperature, which can be attributed to biological mechanisms [72]. Therefore, if biotic uptake was relevant in our streams, the relationship with temperature should have been positive, because increases in thermal energy stimulate biotic $P$ uptake [73]. However, we found a negative effect of temperature on P uptake, suggesting lower P uptake at high temperatures. We also found a negative slope in the correlations between SRP $U_{a m b}$ $(B=-4.60)$, as well as $V_{f-a m b}(B=-3.71)$, and $D O$. The negative effects of both higher temperature and DO concentration on P uptake seem contradictory, as low DO saturation occurs at higher temperature. As both higher streamwater DO and temperature should stimulate aerobic biotic P uptake in the advective zone, these negative relationships may also point to the importance of sediment processes, that is, geochemical SRP sorption and SRP release processes [71]. In conclusion, our data suggested that abiotic $\mathrm{P}$ sorption may be a relevant process in our streams.

Among inorganic $\mathrm{N}$-forms, the demand for $\mathrm{NH}_{4}-\mathrm{N}$ was high, while the mass removal of $\mathrm{NO}_{3}-\mathrm{N}$ was greater; $\mathrm{NH}_{4}-\mathrm{N} \mathrm{V} \mathrm{V}_{\text {f-amb }}$ was higher than $\mathrm{NO}_{3}-\mathrm{N} \mathrm{V}_{\mathrm{f}-\mathrm{amb}}$, but $\mathrm{NO}_{3}-\mathrm{N} \mathrm{U}_{\text {amb }}$ was much greater than $\mathrm{NH}_{4}-\mathrm{N} \mathrm{U}_{\text {amb }}$. The persistently high $\mathrm{NH}_{4}-\mathrm{N} \mathrm{V}_{\mathrm{f}-\mathrm{amb}}$ suggested preferential assimilation of $\mathrm{NH}_{4}-\mathrm{N}$ by aquatic biota (bacteria, fungi, and algae) over $\mathrm{NO}_{3}-\mathrm{N}$, which is consistent with studies carried out in the literature [74-76]. In general, these authors observed that consumers track water column $\mathrm{NH}_{4}-\mathrm{N}$ more closely than water column $\mathrm{NO}_{3}-\mathrm{N}$, as a result of the lower energy-demand associated with the assimilation of $\mathrm{NH}_{4}-\mathrm{N}$ than with that of $\mathrm{NO}_{3}-\mathrm{N}$.

Despite the lower demand for $\mathrm{NO}_{3}-\mathrm{N}$ relative to supply, as represented by its uptake velocities, the utilization of $\mathrm{NO}_{3}-\mathrm{N}$ was considerable. Areal uptake rates (U) for $\mathrm{NO}_{3}-\mathrm{N}$ were up to 80 times greater than $\mathrm{U}_{\mathrm{amb}}$ for $\mathrm{NH}_{4}-\mathrm{N}$. This high $\mathrm{U}_{\mathrm{amb}}$ showed that $\mathrm{NO}_{3}-\mathrm{N}$ is an important source of $\mathrm{N}$ in these systems. Some studies $[23,42]$ have related this condition to the generally greater background concentrations of $\mathrm{NO}_{3}-\mathrm{N}$ than those of $\mathrm{NH}_{4}-\mathrm{N}$. For instance, the authors of [15] observed that $\mathrm{NO}_{3}-\mathrm{N}$ concentrations 10- to 1000-fold greater than $\mathrm{NH}_{4}-\mathrm{N}$ reduced $\mathrm{NH}_{4}-\mathrm{N}$ retention to down to three times in streams in New Hampshire, USA. Similarly, the N demand of microorganisms was primarily satisfied by $\mathrm{NO}_{3}-\mathrm{N}$ as a result of its greater availability, that is, $95 \%$ of the total DIN concentrations across our streams.

The relationships between stream nutrient uptake and its concentrations have received much attention in recent studies that also used pulse nutrient additions for kinetic analysis $[16,27,47,77]$. These authors highlighted that the amount of data provided by the TASCC method is useful for kinetic modelling. However, pulsed additions do not represent ambient uptake from a whole-stream 
perspective, because they do not reflect stable-state transport conditions, but rather transient experimental conditions, with rising and falling limbs of breakthrough curves representing different transport processes [2].

We did not observe saturation conditions, that is, no significant fit to the $\mathrm{M}-\mathrm{M}$ model was found across streams, which was expected because there was no evidence of chronical nutrient enrichment. In some cases, the lack of saturation suggests the existence of a mass transfer component, high-saturation sorption kinetics, or even the occurrence of dissimilatory processes such as nitrification and denitrification that may only saturate at very high concentrations [17]. Our study did not address the specific processes that dominate nutrient uptake, but demonstrated the dominance of biotic nitrate uptake and suggested the importance of abiotic SRP uptake. First-order responses to experimental nutrient enrichment or seasonal or spatial variation in nutrient concentration are commonly observed in streams with low to moderate nutrient concentrations [43], but efficiency loss and especially saturation are the typical responses of streams with chronic nutrient inputs. High streamwater nutrient concentrations due to agricultural and urban land use have been reported for tropical regions [37]. Across 35 highly urbanized tropical watersheds, $\mathrm{NO}_{3}-\mathrm{N}$ and $\mathrm{NH}_{4}-\mathrm{N}$ concentrations as high as 0.41 and $4.42 \mathrm{mg} \cdot \mathrm{L}^{-1}$ have been reported [36]. Similar studies in other tropical streams with chronic nutrient loading would advance our understanding about the potential occurrence of nutrient saturation in tropical streams. Further, more nutrient uptake data is required to understand uptake as function of concentration across a wide variety of tropical streams and rivers, including assessments of abiotic versus biotic uptake, and limitation by mass transfer.

In our study, the uptake of different nutrients and nutrient forms appeared to be tightly associated. All nutrient uptake velocities were positively and strongly correlated with each other, which could suggest the occurrence of co-limitation in our streams [30]. The role of co-limitation in nutrient utilization by microorganisms has been widely investigated in recent studies $[19,28,29,78]$, but had been rarely investigated in tropical stream ecology (but see [19]). As nutrient limitation is an important driver of nutrient uptake [79-81], future investigations should aim at more detailed assessments on how anthropogenic impacts on tropical streams are related to their nutrient limitation and co-limitation conditions. Finally, the relatively high uptake rates observed in the studied tropical Cerrado woodland streams highlight the importance of preservation of these headwater streams for the management of ecosystem functioning and services of Cerrado, and potentially other tropical catchments.

\section{Conclusions}

Here, we evaluated the variability in whole-stream uptake of three important nutrient forms, which is, $\mathrm{SRP}, \mathrm{NH}_{4}-\mathrm{N}$, and $\mathrm{NO}_{3}-\mathrm{N}$, in relatively undisturbed, tropical woodland streams, and found relatively high and coupled uptake rates of these nutrients. Relationships between $\mathrm{NO}_{3}-\mathrm{N}, \mathrm{NH}_{4}-\mathrm{N}$, and SRP uptake in these streams seemed to be due to rather complex interactions of biotic $\mathrm{P}$ limitation, abiotic $\mathrm{P}$ cycling processes, and the preferential uptake of $\mathrm{NH}_{4}-\mathrm{N}$ among $\mathrm{N}$-forms. Drivers of nutrient uptake were partially nutrient form-specific and included unexpected effects, such as negative temperature dependencies of SRP uptake and negative relationships between the uptake of all nutrient forms and SRP concentration. Thus, global change effects on these tropical streams, such as temperature increases due to climate warming and decreased shading as a result of riparian clear-cutting, as well as nutrient enrichment due to urban and agricultural expansion, may have adverse and partially unpredictable impacts on whole-stream nutrient processing, and thus catchment biogeochemistry. Future studies should investigate the mechanisms of nutrient spiraling in tropical streams, such as abiotic versus biotic uptake processes, mass transfer limitation, and nutrient limitation and co-limitation, and how human activities influence them.

Supplementary Materials: The following are available online at http:/ /www.mdpi.com/2073-4441/10/8/1080/ $\mathrm{s} 1$, Table S1: Spearman's correlation coefficients between ambient uptake metrics $\left(\mathrm{S}_{\mathrm{w}-\mathrm{amb}}, \mathrm{U}_{\mathrm{amb}}\right.$, and $\left.\mathrm{V}_{\mathrm{f}-\mathrm{amb}}\right)$, nutrient concentrations and ratios, and in-stream physical and chemical variables. Significant correlations are highlighted in bold $(p<0.05)$, Table S2: Statistical parameters of Michaelis-Menten models used to evaluate the 
relationship between total dynamic uptake rate $\left(\mathrm{U}_{\text {tot-dyn }}\right)$ or total dynamic uptake velocity $\left(\mathrm{V}_{\mathrm{f} \text {-tot-dyn }}\right)$, and total dynamic nutrient concentration $\left(C_{\text {tot-dyn }}\right)$ for both streams. For the Michaelis-Menten model, the maximum uptake rate $\left(\mathrm{U}_{\max } ; \mu \mathrm{g} \cdot \mathrm{m}^{-2} \cdot \mathrm{min}^{-1}\right)$ and the half saturation constant $\left(\mathrm{K}_{\mathrm{m}} ; \mu \mathrm{g} \cdot \mathrm{L}^{-1}\right)$ are shown. The adjusted $\mathrm{R}^{2}$ and $p$-value of fits are reported in brackets, Table S3: Statistical parameters of the efficiency-loss model $\left(\mathrm{U}^{-} \mathrm{aN}^{\mathrm{b}}\right) \mathrm{used}$ to evaluate the fit between the relationship between total dynamic uptake rate $\left(U_{\text {tot-dyn }}\right)$, total dynamic uptake velocity $\left(\mathrm{V}_{\mathrm{f} \text {-tot-dyn }}\right)$, and total dynamic nutrient concentration $\left(\mathrm{C}_{\text {tot-dyn }}\right)$ for both streams. For the efficiency-loss model, $a$ represents the intercept and $b$ is the exponent. The adjusted $\mathrm{R}^{2}$ and $\mathrm{p}$-value of the fits are also reported in brackets, Table S4: Statistical parameters of the first-order model $(U=a+b N)$ used to evaluate the fit between the relationship between total dynamic uptake rate $\left(\mathrm{U}_{\text {tot-dyn }}\right)$, total dynamic uptake velocity $\left(\mathrm{V}_{\mathrm{f} \text {-tot-dyn }}\right)$, and total dynamic nutrient concentration $\left(\mathrm{C}_{\mathrm{tot}-\mathrm{dyn}}\right)$ for both streams. For the efficiency-loss model, $a$ represents the intercept and $b$ is the slope. The adjusted $\mathrm{R}^{2}$ and $p$-value of the fits are also reported in brackets.

Author Contributions: N.R.F and D.G.F.C. designed the framework of the study, conducted fieldwork and analyzed data. N.R.F. coordinated analysis of dissolved nutrients of the samples, and also the modelling of nutrient uptake metrics. I.G.B. and B.G. revised statistical analysis. All authors discussed the results and commented on the manuscript.

Funding: This study was supported by FAPESP (São Paulo Research Foundation, Grant 2016/14176-1) and CNPq Productivity Grant 300899/2016-5). NSF Macrosystems Grant 1442595 supported F.T.

Acknowledgments: N.R.F. thanks CNPq (National Council for Scientific and Technological Development) for scholarship. Special thanks to M.S. Ferreira (University of São Paulo, Brazil), who provided help with mapping and A. Schechner (Kansas State University, USA) for editing the manuscript.

Conflicts of Interest: The authors declare no conflict of interest.

\section{References}

1. Newbold, J.D.; O’Neill, R.V.; Elwood, J.W.; Van Winkle, W. Nutrient Spiraling in Streams: Implications for Nutrient Limitation and Invertebrate Activity. Am. Nat. 1982, 120, 628-652. [CrossRef]

2. Stream Solute Workshop. Concepts and Methods for Assessing Solute Dynamics in Stream Ecosystems. J. N. Am. Benthol. Soc. 1990, 9, 95-119. [CrossRef]

3. Rabalais, N.N. Nitrogen in Aquatic Ecosystems. AMBIO J. Hum. Environ. 2002, 31, 102-112. [CrossRef]

4. Vanni, M.J.; Renwick, W.H.; Bowling, A.M.; Horgan, M.J.; Christian, A.D. Nutrient stoichiometry of linked catchment-lake systems along a gradient of land use. Freshw. Biol. 2011, 56, 791-811. [CrossRef]

5. Matson, P.A.; McDowell, W.H.; Townsend, A.R.; Vitousek, P.M. The globalization of N deposition: Ecosystem consequences in tropical environments. Biogeochemistry 1999, 46, 67-83. [CrossRef]

6. Bennett, E.M.; Carpenter, S.R.; Caraco, N.F. Human Impact on Erodable Phosphorus and Eutrophication: A Global Perspective. AIBS Bull. 2001, 51, 227-231. [CrossRef]

7. Galloway, J.N.; Townsend, A.R.; Erisman, J.W.; Bekunda, M.; Cai, Z.; Freney, J.R.; Martinelli, L.A.; Seitzinger, S.P.; Sutton, M.A. Transformation of the Nitrogen Cycle: Recent Trends, Questions, and Potential Solutions. Science 2008, 320, 889-892. [CrossRef] [PubMed]

8. Elser, J.J.; Bracken, M.E.S.; Cleland, E.E.; Gruner, D.S.; Harpole, W.S.; Hillebrand, H.; Ngai, J.T.; Seabloom, E.W.; Shurin, J.B.; Smith, J.E. Global analysis of nitrogen and phosphorus limitation of primary producers in Freshwater, marine and terrestrial ecosystems. Ecol. Lett. 2007, 10, 1135-1142. [CrossRef] [PubMed]

9. Hall, R.J.O.; Tank, J.L. Ecosystem metabolism controls nitrogen uptake in streams in Grand Teton National Park, Wyoming. Limnol. Oceanogr. 2003, 48, 1120-1128. [CrossRef]

10. Fellows, C.S.; Valett, H.M.; Dahm, C.N.; Mulholland, P.J.; Thomas, S.A. Coupling nutrient uptake and energy flow in headwater streams. Ecosystems 2006, 9, 788-804. [CrossRef]

11. Gücker, B.; Pusch, M.T. Regulation of nutrient uptake in eutrophic lowland streams. Limnol. Oceanogr. 2006, 51, 1443-1453. [CrossRef]

12. Valett, H.M.; Crenshaw, C.L.; Wagner, P.F.; Oct, N. Stream Nutrient Uptake, Forest Succession and Biogeochemical Theory. Ecology 2002, 83, 2888-2901. [CrossRef]

13. Gücker, B.; Boëchat, I. Stream morphology controls ammonium retention. Ecology 2004, 85, $2818-2827$. [CrossRef]

14. Ensign, S.H.; Doyle, M.W. Nutrient spiraling in streams and river networks. J. Geophys. Res. Biogeosci. 2006, 111, 1-13. [CrossRef] 
15. Bernhardt, E.S.; Hall, R.O.; Likens, G.E. Whole-system estimates of nitrification and nitrate uptake in streams of the Hubbard Brook Experimental Forest. Ecosystems 2002, 5, 419-430. [CrossRef]

16. Rodríguez-Cardona, B.; Wymore, A.S.; McDowell, W.H. DOC: $\mathrm{NO}_{3}{ }^{-}$ratios and $\mathrm{NO}_{3}{ }^{-}$uptake in forested headwater streams. J. Geophys. Res. Biogeosci. 2016, 121, 205-217. [CrossRef]

17. Dodds, W.K.; López, A.J.; Bowden, W.B.; Gregory, S.; Grimm, N.B.; Hamilton, S.K.; Hershey, A.E.; Martí, E.; McDowell, W.H.; Meyer, J.L.; et al. N uptake as a function of concentration in streams. J. N. Am. Benthol. Soc. 2002, 21, 206-220. [CrossRef]

18. Earl, S.R.; Valett, H.M.; Webster, J.R. Nitrogen saturation in stream ecosystems. Ecology 2006, 87, 3140-3151. [CrossRef]

19. Tromboni, F.; Thomas, S.A.; Gücker, B.; Neres-Lima, V.; Lourenço-Amorim, C.; Moulton, T.P.; Silva-Junior, E.F.; Feijó-Lima, R.; Boëchat, I.G.; Zandonà, E. Nutrient limitation and the stoichiometry of nutrient uptake in a tropical rainforest stream. J. Geophys. Res. Biogeosci. 2018. [CrossRef]

20. O'Brien, J.M.; Dodds, W.K.; Wilson, K.C.; Murdock, J.N.; Eichmiller, J. The saturation of N cycling in Central Plains streams: $15 \mathrm{~N}$ experiments across a broad gradient of nitrate concentrations. Biogeochemistry 2007, 84, 31-49. [CrossRef]

21. Hall, R.O.; Bernhardt, E.S.; Likens, G.E. Relating Nutrient Uptake with Transient Storage in Forested Mountain Streams. Limnol. Oceanogr. 2008, 47, 255-265. [CrossRef]

22. Martí, E.; Fonollà, P.; Von Schiller, D.; Sabater, F.; Argerich, A.; Ribot, M.; Riera, J.L. Variation in stream C, N and $\mathrm{P}$ uptake along an altitudinal gradient: A space-for-time analogue to assess potential impacts of climate change. Hydrol. Res. 2009, 40, 123. [CrossRef]

23. Ribot, M.; Von Schiller, D.; Peipoch, M.; Sabater, F.; Grimm, N.B.; Martí, E. Influence of nitrate and ammonium availability on uptake kinetics of stream biofilms. Freshw. Sci. 2013, 32, 1155-1167. [CrossRef]

24. Hoellein, T.J.; Tank, J.L.; Rosi-Marshall, E.J.; Entrekin, S.A.; Lamberti, G.A. Controls of nutrient variation on spatial and temporal variation of uptake in three Michigan headwater streams. Limnology 2007, 52, 1964-1977. [CrossRef]

25. Von Schiller, D.; Martí, E.; Riera, J.L.; Ribot, M.; Argerich, A.; Fonolla, P.; Sabater, F. Inter-annual, Annual, and Seasonal Variation of $\mathrm{P}$ and $\mathrm{N}$ Retention in a Perennial and an Intermittent Stream. Ecosystems 2008, 11, 670-687. [CrossRef]

26. Bechtold, H.A.; Marcarelli, A.M.; Baxter, C.V.; Inouye, R.S. Effects of N, P, and organic carbon on stream biofilm nutrient limitation and uptake in a semi-arid watershed. Limnol. Oceanogr. 2012, 57, 1544-1554. [CrossRef]

27. Piper, L.R.; Cross, W.F.; McGlynn, B.L. Colimitation and the coupling of N and P uptake kinetics in oligotrophic mountain streams. Biogeochemistry 2017, 132, 165-184. [CrossRef]

28. Hill, B.H.; Mccormick, F.H.; Harvey, B.C.; Johnson, S.L.; Warren, M.L.; Elonen, C.M. Microbial enzyme activity, nutrient uptake and nutrient limitation in forested streams. Freshw. Biol. 2010, 55, 1005-1019. [CrossRef]

29. Schade, J.D.; Macneill, K.; Thomas, S.A.; Camille Mcneely, F.; Welter, J.R.; Hood, J.; Goodrich, M.; Power, M.E.; Finlay, J.C. The stoichiometry of nitrogen and phosphorus spiralling in heterotrophic and autotrophic streams. Freshw. Biol. 2011, 56, 424-436. [CrossRef]

30. Gibson, C.A.; Reilly, C.M.O.; Conine, A.L.; Lipshutz, S.M. Nutient uptake across a gradient of nutrient concentration and ratios at the landscape scale. J. Geophys. Res. Biogeosci. 2015, 120, 326-340. [CrossRef]

31. Wymore, A.S.; Coble, A.A.; Rodríguez-Cardona, B.; McDowell, W.H. Nitrate uptake across biomes and the influence of elemental stoichiometry: A new look at LINX II. Glob. Biogeochem. Cycles 2016, 30, 1183-1191. [CrossRef]

32. Schlesinger, W.H.; Cole, J.J.; Finzi, A.C.; Holland, E.A. Introduction to coupled biogeochemical cycles. Front. Ecol. Environ. 2011, 9, 5-8. [CrossRef]

33. Marklein, A.R.; Houlton, B.Z. Nitrogen inputs accelerate phosphorus cycling rates across a wide variety of terrestrial ecosystems. New Phytol. 2012, 193, 696-704. [CrossRef] [PubMed]

34. UNFPA. The State of World Population. 2017. Available online: https://www.unfpa.org/sites/default/ files/sowp/downloads/UNFPA_PUB_2017_EN_SWOP.pdf (accessed on 1 August 2018).

35. MEA. Millennium Ecosystem Assessment. Ecosystems and Human Well Being: Synthesis; Island Press: Washington, DC, USA, 2005. 
36. Tromboni, F.; Dodds, W.K. Relationships between Land Use and Stream Nutrient Concentrations in a Highly Urbanized Tropical Region of Brazil: Thresholds and Riparian Zones. Environ. Manag. 2017, 60, 30-40. [CrossRef] [PubMed]

37. Gücker, B.; Silva, R.C.S.; Graeber, D.; Monteiro, J.A.F.; Brookshire, E.N.J.; Chaves, R.C.; Boëchat, I.G. Dissolved nutrient exports from natural and human-impacted Neotropical catchments. Glob. Ecol. Biogeogr. 2016, 25, 378-390. [CrossRef]

38. Mulholland, P.J.; Tank, J.L.; Webster, J.R.; Bowden, W.B.; Dodds, W.K.; Gregory, S.V.; Grimm, N.B.; Hamilton, S.K.; Johnson, S.L.; Mart, E.; et al. Can uptake length in streams be determined by nutrient addition experiments? Results from an interbiome comparison study. J. N. Am. Benthol. Soc. 2002, 21, 544-560. [CrossRef]

39. Solomon, C.T.; Hotchkiss, E.R.; Moslemi, J.M.; Ulseth, A.J.; Stanley, E.H.; Hall, R.O., Jr.; Flecker, A.S. Sediment size and nutrients regulate denitrification in a tropical stream. J. N. Am. Benthol. Soc. 2009, 29, 480-490. [CrossRef]

40. Potter, J.D.; McDowell, W.H.; Merriam, J.L.; Peterson, B.J.; Thomas, S.M. Denitrification and total nitrate uptake in streams of a tropical landscape. Ecol. Appl. 2010, 20, 2104-2115. [CrossRef] [PubMed]

41. Koenig, L.E.; Song, C.; Wollheim, W.M.; Rüegg, J.; Mcdowell, W.H. Nitrification increases nitrogen export from a tropical river network. Freshw. Sci. 2017, 36, 698-712. [CrossRef]

42. Newbold, J.D.; Bott, T.L.; Kaplan, L.A.; Dow, C.L.; Jackson, J.K.; Aufdenkampe, A.K.; Martin, L.A.; Van Horn, D.J.; de Long, A.A. Uptake of nutrients and organic C in streams in New York City drinking-water-supply watersheds. J. N. Am. Benthol. Soc. 2006, 25, 998-1017. [CrossRef]

43. Mulholland, P.J.; Helton, A.M.; Poole, G.C.; Hall, R.O.; Hamilton, S.K.; Peterson, B.J.; Tank, J.L.; Ashkenas, L.R.; Cooper, L.W.; Dahm, C.N.; et al. Stream denitrification across biomes and its response to anthropogenic nitrate loading. Nature 2008, 452, 202-205. [CrossRef] [PubMed]

44. Gibson, C.A.; O'Reilly, C.M. Organic matter stoichiometry influences nitrogen and phosphorus uptake in a headwater stream. Freshw. Sci. 2012, 31, 395-407. [CrossRef]

45. Bernot, M.J.; Tank, J.L.; Royer, T.V.; David, M.B. Nutrient uptake in streams draining agricultural catchments of the midwestern United States. Freshw. Biol. 2006, 51, 499-509. [CrossRef]

46. Niyogi, D.K.; Simon, K.S.; Townsend, C.R. Land use and stream ecosystem functioning: Nutrient uptake in streams that contrast in agricultural development. Arch. Hydrobiol. 2004, 160, 471-486. [CrossRef]

47. Covino, T.; McGlynn, B.; McNamara, R. Land use/land cover and scale influences on in-stream nitrogen uptake kinetics. J. Geophys. Res. Biogeosci. 2012, 117, 1-13. [CrossRef]

48. Fonseca, B.M.; De Mendonça-Galvão, L.; Padovesi-Fonseca, C.; De Abreu, L.M.; Fernandes, A.C.M. Nutrient baselines of Cerrado low-order streams: Comparing natural and impacted sites in Central Brazil. Environ. Monit. Assess. 2014, 186, 19-33. [CrossRef] [PubMed]

49. Covino, T.P.; McGlynn, B.L.; McNamara, R.A. Tracer Additions for Spiraling Curve Characterization (TASCC): Quantifying stream nutrient uptake kinetics from ambient to saturation. Limnol. Oceanogr. Methods 2010, 8, 484-498. [CrossRef]

50. Solórzano, L. Determination of ammonia in natural waters by phenol hypochlorite method. Limnol. Oceanogr. 1969, 14, 799-801. [CrossRef]

51. APHA. Standard Methods for the Examination of Water and Wastewater, 22nd ed.; American Water Works Assn: Washington, DC, USA, 2012.

52. Lemmon, P.E. A New Instrument for Measuring Forest Overstory Density. J. For. 1957, 55, 667-668.

53. Lemmon, P.E. A Spherical Densiometer for Estimating Forest Overstory Density. For. Sci. 1956, 2, $314-320$.

54. Webster, J.R.; Valett, H.M. Solute Dynamics. Methods in Stream Ecology; Hauer, F.R., Lamberti, G., Eds.; Elsevier Inc.: New York, NY, USA, 2006; pp. 169-185, ISBN 9780123329080.

55. Alexander, R.B.; Smith, R.A.; Schwarz, G.E. Effect of stream channel size on the delivery of nitrogen to the Gulf of Mexico. Nature 2000, 403, 758-762. [CrossRef] [PubMed]

56. Webster, J.R.; Mulholland, P.J.; Tank, J.L.; Valett, H.M.; Dodds, W.K.; Peterson, B.J.; Bowden, W.B.; Dahm, C.N.; Findlay, S.; Gregory, S.V. Others Factors affecting ammonium uptake in streams-an inter-biome perspective. Freshw. Biol. 2003, 48, 1329-1352. [CrossRef]

57. Rosa, R.D.S.; Aguiar, A.C.F.; Boëchat, I.G.; Gücker, B. Impacts of fish farm pollution on ecosystem structure and function of tropical headwater streams. Environ. Pollut. 2013, 174, 204-213. [CrossRef] [PubMed] 
58. Saltarelli, W.A.; Dodds, W.K.; Tromboni, F.; Calijuri, M.C.; Neres-lima, V.; Jordão, C.E.; Cunha, D.G.F. Variation of stream metabolism along a tropical environmental gradient. J. Limnol. 2018. [CrossRef]

59. Merriam, J.L.; McDowell, W.H.; Tank, J.L.; Wollheim, W.M.; Crenshaw, C.L.; Johnson, S.L. Characterizing nitrogen dynamics, retention and transport in a tropical rainforest stream using an in situ ${ }^{15} \mathrm{~N}$ addition. Freshw. Biol. 2002, 47, 143-160. [CrossRef]

60. Small, G.E.; Ardón, M.; Duff, J.H.; Jackman, A.P.; Ramírez, A.; Triska, F.J.; Pringle, C.M. Phosphorus retention in a lowland Neotropical stream following an eight-year enrichment experiment. Freshw. Sci. 2016, 35, 1-11. [CrossRef]

61. Schanz, F.; Juon, H. Two different methods of evaluating nutrient limitations of periphyton bioassays, using water from the River Rhine and eight of its tributaries. Hydrobiologia 1983, 102, 187-195. [CrossRef]

62. Dodds, W.; Whiles, M. Nitrogen, sulfur, phosphorus, and other nutrients. In Freshwater Ecology; Dodds, W.K., Whiles, M., Eds.; Elsevier Inc.: Amsterdam, The Netherlands, 2010; ISBN 978-0-12-374724-2.

63. Newbold, J.D.; Elwood, J.W.; O’Neill, R.V.; Sheldon, A.L. Phosphorus Dynamics in a Woodland Stream Ecosystem: A Study of Nutrient Spiralling. Ecology 1983, 64, 1249-1265. [CrossRef]

64. Meyer, J.L. The Role of Sediments and Bryophytes in Phosphorus Dynamics in a Head Water Stream Ecosystem. Limnol. Oceanogr. 1979, 24, 365-375. [CrossRef]

65. Demars, B.O.L. Whole-stream phosphorus cycling: Testing methods to assess the effect of saturation of sorption capacity on nutrient uptake length measurements. Water Res. 2008, 42, 2507-2516. [CrossRef] [PubMed]

66. Powers, S.M.; Stanley, E.H.; Lottig, N.R. Quantifying phosphorus uptake using pulse and steady-state approaches in streams. Limnol. Oceanogr. Methods 2009, 7, 498-508. [CrossRef]

67. House, W.A.; Warwick, M.S. Interactions of phosphorus with sediments in the River Swale, Yorkshire, UK. Hydrol. Process. 1999, 13, 1103-1115. [CrossRef]

68. Reddy, K.R.; Kadlec, R.H.; Flaig, E.; Gale, P.M. Phosphorus retention in streams and wetlands: A review. Crit. Rev. Environ. Sci. Technol. 1999, 29, 83-146. [CrossRef]

69. House, W.H.; Denison, F.H.; Armitage, P. Comparison of the uptake of inorganic P to a suspended and stream bed-sediment. Water Res. 1995, 29, 767-779. [CrossRef]

70. Silva, J.S.O.; da Bustamante, M.M.C.; Markewitz, D.; Krusche, A.V.; Ferreira, L.G. Effects of land cover on chemical characteristics of streams in the Cerrado region of Brazil. Biogeochemistry 2011, 105, 75-88. [CrossRef]

71. Mortimer, C.H. Chemical exchanges betweeen sediments and water in the Great Lakes-Speculations on probable regulatory mechanisms. Limnol. Oceanogr. 1971, 16, 387-404. [CrossRef]

72. Angelo, A.D.J.D.; Webster, J.R.; Benfield, E.F. Mechanisms of Stream Phosphorus Retention: An Experimental Study. J. N. Am. Benthol. Soc. 1991, 10, 225-237. [CrossRef]

73. Valett, H.M.; Thomas, S.A.; Mulholland, P.J.; Webster, J.R.; Dahm, C.N.; Fellows, C.S.; Crenshaw, C.L.; Peterson, C.G. Endogenous and exogenous control of ecosystem function: N cycling in headwater streams. Ecology 2008, 89, 3515-3527. [CrossRef] [PubMed]

74. Dodds, W.K.; Priscu, J.C.; Ellis, B.K. Seasonal uptake and regeneration of inorganic nitrogen and phosphorus in a large oligotrophic lake: Size-fractionation and antibiotic treatment. J. Plankton Res. 1991, 13, 1339-1358. [CrossRef]

75. Pastor, A.; Peipoch, M.; Cañas, L.; Chappuis, E.; Ribot, M.; Gacia, E.; Riera, J.L.; Martí, E.; Sabater, F. Nitrogen stable isotopes in primary uptake compartments across streams differing in nutrient availability. Environ. Sci. Technol. 2013, 47, 10155-10162. [CrossRef] [PubMed]

76. Oviedo-Vargas, D.; Royer, T.V.; Johnson, L.T. Dissolved organic carbon manipulation reveals coupled cycling of carbon, nitrogen, and phosphorus in a nitrogen-rich stream. Limnol. Oceanogr. 2013, 58, 1196-1206. [CrossRef]

77. Diemer, L.A.; McDowell, W.H.; Wymore, A.S.; Prokushkin, A.S. Nutrient uptake along a fire gradient in boreal streams of Central Siberia. Freshw. Sci. 2015, 34, 1443-1456. [CrossRef]

78. Appling, A.P.; Heffernan, J.B. Nutrient Limitation and Physiology Mediate the Fine-Scale (De)coupling of Biogeochemical Cycles. Am. Nat. 2014, 184, 384-406. [CrossRef] [PubMed]

79. Tank, J.L.; Reisinger, A.J. Nutrient Limitation and Uptake Rates in Streams and Rivers of the Greater Yellowstone Ecosystem. Univ. Wyoming Natl. Park Serv. Res. Cent. Annu. Rep. 2013, 36, 153-159. 
80. King, S.A.; Heffernan, J.B.; Cohen, M.J. Nutrient flux, uptake, and autotrophic limitation in streams and rivers. Freshw. Sci. 2014, 33, 85-98. [CrossRef]

81. Jarvie, H.P.; Smith, D.R.; Norton, L.R.; Edwards, F.K.; Bowes, M.J.; King, S.M.; Scarlett, P.; Davies, S.; Dils, R.M.; Bachiller-Jareno, N. Phosphorus and nitrogen limitation and impairment of headwater streams relative to rivers in Great Britain: A national perspective on eutrophication. Sci. Total Environ. 2018, 621, 849-862. [CrossRef] [PubMed] 